\title{
Association of FOXM1 expression with tumor histology and prognosis in Wilms tumor: Potential for a new prognostic marker
}

\author{
NADJA APELT ${ }^{1}$, JOCHEN HUBERTUS $^{1}$, DORIS MAYR $^{2}$, NORBERT GRAF $^{3}$, \\ RHOIKOS FURTWÄNGLER ${ }^{3}$, DIETRICH VON SCHWEINITZ ${ }^{1}$ and ROLAND KAPPLER ${ }^{1}$
}

${ }^{1}$ Department of Pediatric Surgery, Dr. von Hauner Children's Hospital; ${ }^{2}$ Institute of Pathology, Ludwig Maximilian University of Munich, Munich, 80337 Bavaria; ${ }^{3}$ Department of Pediatric Oncology and Hematology, University of Saarland, Homburg, 66421 Saarland, Germany

Received July 13, 2015; Accepted April 22, 2016

DOI: $10.3892 / \mathrm{ol} .2016 .4958$

\begin{abstract}
Wilms tumor (WT) is the most common pediatric renal malignancy. A recent ontogenic model suggests that undifferentiated tumor state, and hence poor prognosis, in WT is determined by stabilization of $\beta$-catenin in the nucleus. Forkhead box M1 (FOXM1) is a downstream component of the Wnt pathway and promotes nuclear localization of $\beta$-catenin. As elevation of FOXM1 gene expression is prognostic in various types of malignancy, we hypothesized that high FOXM1 expression in WT is associated with undifferentiated histology and thus poor prognosis. In the current study, the expression of FOXM1 mRNA was determined in $46 \mathrm{WT}$ specimens and 11 renal tissue controls from patients undergoing tumor nephrectomy, and these data were assessed with regard to clinicopathological parameters. The results demonstrated an upregulation of FOXM1 in WT by 10-fold compared to normal tissue. Expression differed significantly between controls and tumors of intermediate- and high-risk histopathology ( $\mathrm{P}<0.001$, Kruskal-Wallis), and distinguished normal tissue from tumors of good and adverse clinical outcome ( $\mathrm{P}<0.001$, Kruskal-Wallis). Notably, FOXM1 expression was significantly lower $(\mathrm{P}=0.009)$ in patients that received preoperative doxorubicin. These results suggest that FOXM1 may serve as a companion diagnostic factor for doxorubicin-based therapies in WT.
\end{abstract}

Correspondence to: Professor Dr Roland Kappler, Department of Pediatric Surgery, Dr von Hauner Children's Hospital, Ludwig Maximilian University of Munich, Lindwurmstraße 2a, Munich, 80337 Bavaria, Germany

E-mail: roland.kappler@med.uni-muenchen.de

Abbreviations: WT, Wilms tumor; SD, standard deviation; CI, confidence interval; SIOP, International Society of Pediatric Oncology

Key words: Wilms tumor, FOXM1, forkhead box, prognosis, nephroblastoma

\section{Introduction}

Wilms tumor (WT) is the most common pediatric renal malignancy, affecting $\sim 7$ in 100,000 children (1). Therapy is efficacious with excellent cure rates, yielding a $>90 \%$ 5 -year overall survival rate (2). However, the survival times of patients with high-risk histology nephroblastoma (i.e. blastemal predominant subtype following chemotherapy or diffuse anaplasia) or metastatic disease remain limited. Despite significant progress in developing risk predictors for disease recurrence, current molecular and epigenetic markers do not allow the reliable identification and preemptive treatment of all high-risk patients. Those patients who develop recurrent disease continue to suffer a 50\% mortality rate, even after salvage therapy (2). Thus, an improved understanding of WT tumorigenesis may indeed be useful in identifying new therapeutic targets, and also more reliable prognostic markers.

Current models of Wilms tumorigenesis stress the pivotal role of canonical Wnt signaling aberrations in tumor stem cell development and tumor survival before the eighth week of gestation $(3,4)$. These ontogenetic models propose a 'two-hit' hypothesis wherein disrupted Wnt signaling is the necessary adjunct to genetic (WT1, CTNNB1, WTX) (4) and epigenetic [loss of imprinting (LOI) at chromosome 11p] $(2,5,6)$ aberrations of the tumor stem cell. In brief, extracellular Wnt ligands trigger a signaling cascade that culminates in the translocation of cytoplasmic $\beta$-catenin into the nucleus. There, $\beta$-catenin induces target genes that cause the cell to form pre-tubular aggregates (4). Notably, $\beta$-catenin degradation is important for further epithelial differentiation of the cell into nephron tissue. Should $\beta$-catenin be stabilized in the nucleus, pre-tubular aggregate cells escape apoptosis and undergo proliferation, while cellular epithelial transition is fully blocked (4). Thus, the stabilization of $\beta$-catenin in the nucleus is theorized to be essential in the formation of nephrogenic blastemal rests and presumed WT stem cells. It is thought to be involved in the tumorigenesis of all WT, including those without mutations (4). Alterations in Wnt signaling have been described in all five subtypes (S1-S5) of a proposed new ontogenic model of Wilms tumorigenesis, which groups tumors by 
gene expression, mutation analysis, methylation analysis and canonical Wnt activation (3).

Forkhead box (FOX) proteins are DNA-binding factors that regulate transcription and DNA repair. Their role in embryogenesis in addition to tumorigenesis has received great attention (7-9). Notably, FOXM1 has been reported to be a critical downstream component of Wnt signaling in glioma cells, where it enhances and stabilizes $\beta$-catenin in the nucleus, forming a transcription/activation complex with $\beta$-catenin (10). Previous work suggests that FOXM1 is essential for maintaining an undifferentiated tumor state, thus promoting tumorigenesis $(8,10)$. Indeed, spontaneous differentiation of neuroblastoma cells upon FOXM1 ablation has been demonstrated (10). Furthermore, FOXM1 has been implicated in cell migration, invasion, angiogenesis and metastasis by upregulating vascular endothelial growth factor and molecules of cell-cycle progression, such as cyclin B1, aurora kinase B, polo-like kinase 1 and c-Myc $(7,8)$. FOXM1 was demonstrated to be substantially elevated in numerous types of human tumor, where it seems to protect cells from senescence and reactive oxygen species $(8,9)$.

Given the outstanding importance attributed to the stabilization of $\beta$-catenin in the nucleus for Wilms tumorigenesis $(3,4)$, as well as the recent discovery that FOXM1 is a downstream component of Wnt signaling (10), we hypothesized that FOXM1 is critical in Wilms tumorigenesis and may affect patient outcome. To test this hypothesis, FOXMI expression in WT was measured and its impact on outcome was assessed in the present study.

\section{Materials and methods}

Study population. A total of $46 \mathrm{WT}$ specimens were investigated, following cryopreservation in liquid nitrogen, from patients undergoing surgical tumor resection at the Department of Pediatric Surgery of the Dr. von Hauner Children's Hospital (Munich, Germany). Only one sample per tumor was analyzed, and analysis included all tumor samples available to us. Likewise, for bilateral cases, one sample taken from the kidney with the larger tumor mass was analyzed per patient. The median age at the time of surgery was 37.6 months (range, 0 months to 17 years), with a female:male gender ratio of 1:1.9. All patients were treated according to the International Society of Pediatric Oncology (SIOP) protocol (11) and underwent chemotherapy prior to surgery. Stage 1 disease was identified in 16 patients $(34.8 \%)$. A further 16 patients $(34.8 \%)$ were found to have bilateral WT. The control group $(n=11)$ consisted of renal tissue from the healthy part of the resected specimen following tumor nephrectomy. There were 3 controls taken from patients with stage 1 disease, and another 4 from patients suffering bilateral WT. The median age of the control group was 36.9 months (range, 2-62 months) with a female:male ratio of 1:1.8 (Table I). Histological classification of each sample was performed by a trained pathologist prior to nucleic acid isolation. The study was approved by the ethics committee of the Ludwig Maximilian University of Munich. Written consent was obtained from all parents.

Reverse transcription (RT)-quantitative polymerase chain reaction ( $q P C R$ ). TRI Reagent ${ }^{\circledR}$ (Sigma-Aldrich, Munich,
Germany) was used for the isolation of total RNA from native samples according to the supplier's recommendations. Total RNA was depleted of DNA and subsequently purified using DNase and an RNeasy Mini Kit, respectively (Qiagen $\mathrm{GmbH}$, Hilden, Germany). RT of total RNA was performed using random hexamers (Roche Diagnostics, Penzberg, Germany) and SuperScript II reverse transcriptase (Invitrogen; Thermo Fisher Scientific, Carlsbad, CA, USA). Subsequently, cDNA was analyzed by qPCR on a Mastercycler RealPlex ${ }^{2}$ cycler (Eppendorf, Hamburg, Germany) using iTaq $^{\mathrm{TM}}$ Universal SYBR $^{\circledR}$ Green Supermix (Bio-Rad Laboratories GmbH, Munich, Germany) and the following primer pairs (5'-3' orientation): FOXM1 forward, CTCCCGCAGCATCAAGCAA, and reverse GCCAGGACGCTGATGGTCTC; TATA box-binding protein $(T B P)$ forward, GCCCGAAACGCCGAATAT, and reverse, CCGTGGTTCGTGGCTCTCT. In brief, samples were heated to $95^{\circ} \mathrm{C}$ for $2 \mathrm{~min}$, and subsequently processed over 40 cycles at 95,55 and $68^{\circ} \mathrm{C}$ for 15,15 and $20 \mathrm{sec}$, respectively. TBP was used as a housekeeping gene to standardize the amount of sample RNA. Relative quantization of gene expression was performed using a previously described mathematical model (12).

Methylation status of the insulin-like growth factor 2 (IGF2)/H19 locus. Determination of methylation status was performed using a previously described qPCR-based method $(5,6)$. In brief, genomic DNA was extracted from native tumor samples using standard procedures. DNA was subsequently digested with either RsaI, RsaI+HpaII or MspI for $4 \mathrm{~h}$ at $37^{\circ} \mathrm{C}$. Restricted DNA was then amplified using the following primer pairs (5'-3' orientation): H19DMR forward, GGCCCTAGTGTGAAACCCTTCTCG, and reverse, CAG GCGGTGAGACCGAAGGA; KvDMR forward, CCCGCT GGGCCAATCT, and reverse, GAGTCTGGTTTTGATGC CACC. Subsequently, the generated template DNA was analyzed by qPCR on a Mastercycler RealPlex ${ }^{2}$ cycler as described (5). The amount of amplifiable template remaining after $R s a \mathrm{I}+H p a \mathrm{II}$ digestion was compared to that remaining after single $R s a$ I digest, allowing the percentage of methylation to be estimated. An RsaI+MspI digest was used as a control for complete digestion. The percentage of methylated (undigested) DNA was calculated by dividing the amount of DNA amplified from the RsaI+HpaII digested sample by that obtained from the control RsaI digest.

Data analysis. Data analysis was performed using SPSS software version 21 (IBM SPSS, Armonk, NY, USA). Tests included the one-sample Kolmogorov-Smirnov test of normal distribution, the Kruskal-Wallis test and the Mann-Whitney test.

\section{Results}

A total of 46 histopathologically confirmed WT specimens as well as 11 controls of healthy kidney-tissue were analyzed for FOXM1 expression (Fig. 1). Gene expression did not follow normal distribution in either group $(\mathrm{P}<0.0001$; one-sample Kolmogorov-Smirnov test), and was present at highly elevated FOXM1 levels in the tumors. Overall, a 10 -fold elevation in median FOXM1 expression was detected 
Table I. Overview of demographic data.

\begin{tabular}{|c|c|c|}
\hline Variable & Tumor tissue & Normal tissue \\
\hline Number of samples tested & 46 & 11 \\
\hline Lost to follow-up, n (\%) & $3(6.5)$ & $2(18.2)$ \\
\hline \multicolumn{3}{|l|}{ FOXM1 expression } \\
\hline Median & 1.21 & 0.12 \\
\hline SD & 1.53 & 0.14 \\
\hline $95 \% \mathrm{CI}$ & $1.26-2.18$ & $0.09-0.26$ \\
\hline \multicolumn{3}{|l|}{ Gender } \\
\hline Female, n (\%) & $16(34.8)$ & $4(36.4)$ \\
\hline Male, n (\%) & $30(65.2)$ & $7(63.6)$ \\
\hline Female:male & $1: 1.9$ & $1: 1.8$ \\
\hline \multicolumn{3}{|l|}{ Age at operation } \\
\hline Median & 37.6 months & 36.9 months \\
\hline Range & $0-17$ years & $2-62$ months \\
\hline Relapse, n (\%) & $5(11.6)$ & $1(11.1)$ \\
\hline Mortality from disease, n (\%) & $4(9.3)$ & $1(11.1)$ \\
\hline Histology, n (\%) & $\mathrm{n}=43$ & \\
\hline Blastemal & $15(34.9)$ & n.a. \\
\hline Diffuse and focal anaplastic & $2(4.7)$ & n.a. \\
\hline Epithelial & $7(16.3)$ & n.a. \\
\hline Stromal & $2(4.7)$ & n.a. \\
\hline Other & $17(39.5)$ & n.a. \\
\hline SIOP stage, n (\%) & $\mathrm{n}=43$ & $\mathrm{n}=9$ \\
\hline 1 & $16(37.2)$ & $3(33.3)$ \\
\hline 2 & $3(7.0)$ & $0(0.0)$ \\
\hline 3 & $6(14.0)$ & $2(22.2)$ \\
\hline 4 & $3(7.0)$ & $0(0.0)$ \\
\hline 5 & $15(34.9)$ & $4(44.4)$ \\
\hline Doxorubicin administered, n (\%) & $9(20.9)$ & $3(33.3)$ \\
\hline
\end{tabular}

FOXM1, forkhead box M1; SD, standard deviation; CI, confidence interval; SIOP, International Society of Pediatric Oncology; n.a., not applicable.

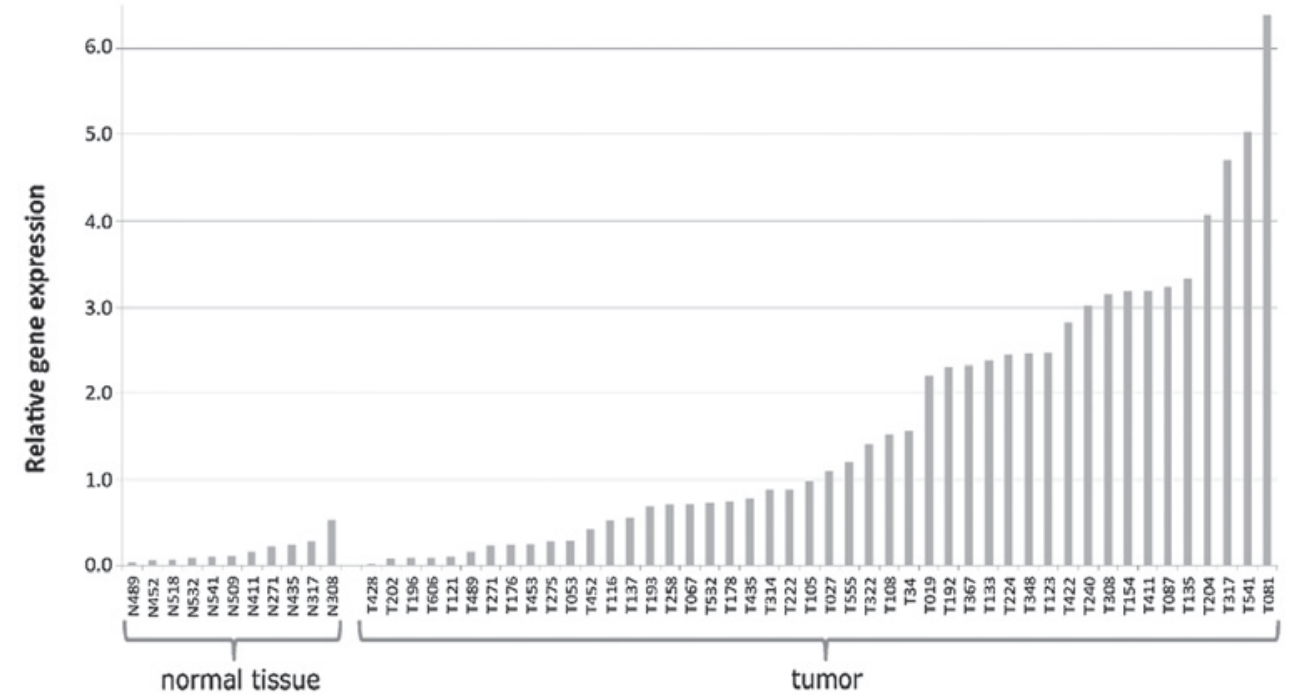

Figure 1. FOXM1 expression in tumor samples and normal tissue. FOXM1 mRNA expression relative to levels of the housekeeping gene TATA box-binding protein in 46 tumor samples and 11 normal tissues. FOXM1, forkhead box M1. 
A

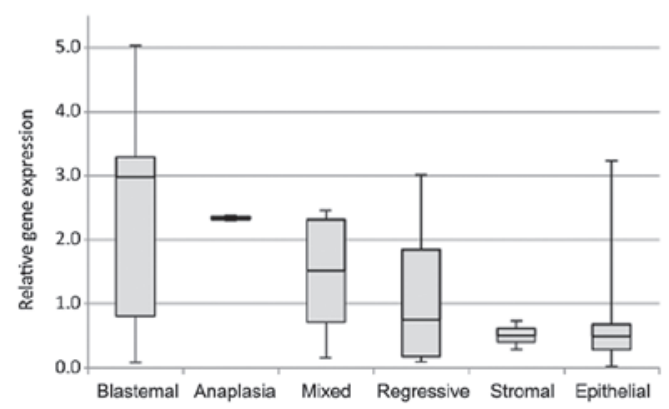

C

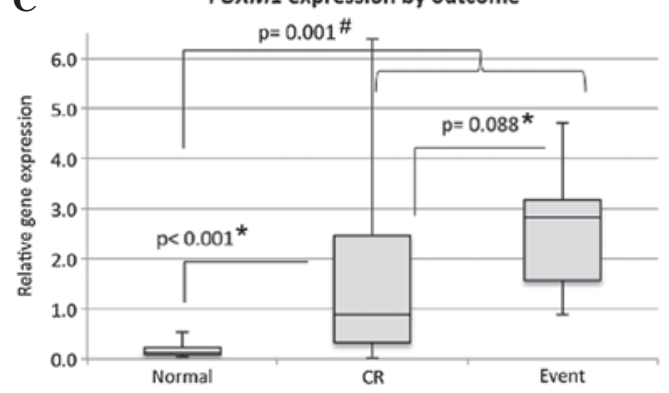

B

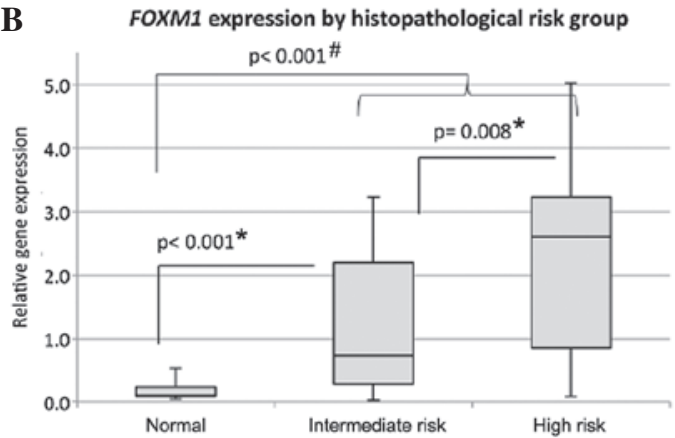

D

FOXM1 expression by SIOP stage

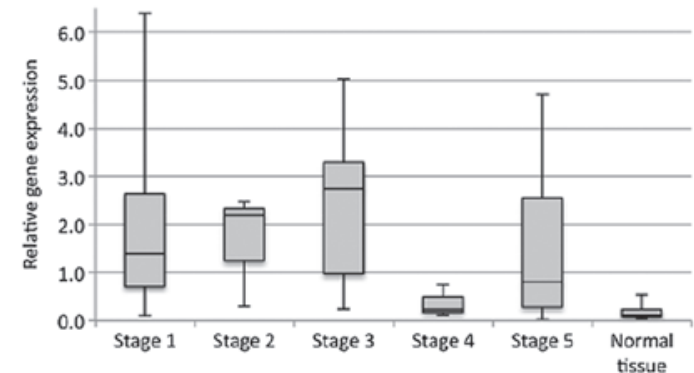

Figure 2. Trends in FOXM1 expression according to histology, risk grouping, outcome and SIOP stage. Variation in FOXM1 expression by (A) tumor histology, and (B) histopathological risk group as defined by SIOP (Intermediate risk: regressive, epithelial, stromal, mixed or focal anaplastic histology. High risk: blastemal or diffuse anaplastic histology). (C) Relative FOXM1 expression by outcome [CR or event (defined as relapse or mortality from disease)]. Statistical differences were calculated with "Mann-Whitney test or ${ }^{\#}$ Kruskal-Wallis. (D) Trend towards higher FOXM1 expression with increasing tumor spread in SIOP stages 1-3 Wilms tumors. Patients with stages 4 and 5 Wilms tumors did receive doxorubicin chemotherapy in the majority of cases. FOXM1, forkhead box M1; SIOP, International Society of Pediatric Oncology; CR, complete remission.

in the tumor samples as compared with the controls (1.21 vs. 0.12; P<0.001; Mann-Whitney U test) (Fig. 1; Table I).

Subsequently, the expression level of tumor cases was assessed with regard to clinicopathological features, which was based only on 43 tumor cases due to the loss of follow-up in 3 patients. FOXM1 expression varied greatly according to histopathological subtype of tumor tissue (Fig. 2A). Blastemal and anaplastic tumors exhibited the highest median values, with relative FOXM1 expression levels of 2.98 [standard deviation (SD), 1.71; 95\% confidence interval (CI), 1.36-3.35] and 2.34 (SD, 0.06; 95\% CI, 1.84-2.84), respectively. Histopathological risk group according to SIOP (11) were significantly correlated $(\mathrm{P}<0.001$, Kruskal-Wallis) with FOXM1 expression: Tumors of high-risk histology (blastemal and diffuse anaplastic histology) exhibited significantly increased median FOXM1 expression compared with tumors of intermediate risk (regressive, epithelial, stromal, mixed or focal anaplastic histology) (2.60 vs. $0.73 ; \mathrm{P}=0.008$, Mann-Whitney U) (Fig. 2B). All 4 mortalities occurred in patients with high-risk histology tumors. Accordingly, a trend towards higher median transcript levels was identified in those tumor samples of patients with events (defined as relapse or mortality from disease); in these, median FOXM1 expression was 2.82 (SD, 1.33; 95\% CI, 1.46-3.80), a >3-fold increase compared with the median of 0.88 (SD 1.51; $95 \%$ CI, 1.49-2.08) identified in tumors of good outcome $(\mathrm{P}=0.088$; Mann-Whitney test) (Fig. 2C).

There was a clear, albeit insignificant (Kruskal-Wallis), trend for increased FOXM1 expression with increasing tumor spread as indicated by SIOP stage (Fig. 2D). Tumors of stages 1, 3 and 5 exhibited significantly elevated median $F O X M 1$ expression levels in comparison to normal tissue $(\mathrm{P}=0.002, \mathrm{P}=0.005$ and $\mathrm{P}=0.002$, respectively; Mann-Whitney U; Fig. 2B), with stage 2 not tested due to insufficient sample size. Stage 4 tumors, all of which were of regressive pathology, proved an exception to this. Patients in this group had received preoperative treatment with doxorubicin and the median FOXM1 expression in this group $(0.24$; SD, $0.28 ; 95 \% \mathrm{CI}, 0.05-0.68)$ was insignificantly higher than in normal tissue $(0.12$; SD, 0.14; 95\% CI, 0.09-0.26).

A significant associated between preoperative therapy with doxorubicin and FOXM1 expression could be observed $(\mathrm{P}<0.001$, Kruskal-Wallis) (Fig. 3A). Significantly lower median FOXM1 expression was identified in stage 5 patients who had received 6 weeks of treatment with actinomycin D, vincristine and doxorubicin when compared to stage 5 patients who had received 6 weeks of vincristine and actinomycin D only [0.42 (SD, 0.99; 95\% CI, 0.06-1.53) vs. 2.45 (SD, 1.19; 95\% CI, 1.52-3.28)]. Notably, prolongation of treatment alone did not have a significant impact on median FOXM1 expression when comparing stage 1 tumors that had received 4 weeks of vincristine and actinomycin D to those stage 5 tumors that had received 6 weeks of treatment without doxorubicin $[1.39$ (SD, 1.63; 95\% CI, 1.12-2.72) vs. 2.45 (SD, 1.19; 95\% CI, 1.52-3.28)] (Fig. 3B). Statistical subgroup analysis regarding histopathology and outcome was not conducted on those 9 patients that received doxorubicin preoperatively, due to insufficient sample size. 
Another known predictor of adverse outcome (5,6), namely loss of heterozygosity ( $\mathrm{LOH}$ ) and LOI aberrations of the IGF2/H19 locus, did not correlate with FOXMI expression ( $\mathrm{P}=0.110$; Kruskal-Wallis), although a trend towards higher FOXM1 expression in tumors with methylation aberrations exists ( $\mathrm{P}=0.064$; Mann-Whitney U) (Fig. 3C).

All other clinical characteristics, including age at surgery and gender, were not significantly associated with FOXM1 expression.

\section{Discussion}

WT is considered a prime example of differentiation failure in human neoplasia (4). Nevertheless, oncogenesis in WT is currently not fully understood. Despite the importance of genetic and epigenetic changes in Wilms tumorigenesis $(5,6)$, stabilization of $\beta$-catenin in the nucleus is theorized to be the essential 'first hit' in a two-hit hypothesis describing the formation of nephrogenic blastemal rests and presumed tumor stem cells $(3,4)$. Recently, FOXM1 was shown to be a downstream component of Wnt signaling, stabilizing $\beta$-catenin in the nucleus of glioma cells (10). Conversely, ablation of FOXM1 was demonstrated to be therapeutic in neuroblastoma and glioma models $(8,10)$, suggesting that, in such tumors, FOXM1 may not only be a potential adjunct biomarker in determining prognosis, but also a promising future target of chemotherapy.

To the best of our knowledge, the current study was the first to demonstrate a 10-fold elevation of FOXM1 expression in WT cells as compared with normal kidney tissue. The fact that no significant correlation was identified between elevated FOXM1 expression and methylation status at the differentially methylated region IGF2/H19 may conceivably support an independent mechanism for such an elevation. Considering the proposed role of FOXM1 as a downstream component of Wnt signaling (10), these results support the ontogenetic models proposed by Pode-Shakked and Dekel (4) and Gadd et al (3), respectively.

Despite recent advances in genomic and epigenetic analysis, tumor histology is still recognized as the most powerful prognostic factor determining survival in pretreated WT (13). Undifferentiated tumor state, as observed in diffuse anaplastic and blastemal WT, is associated with decreased survival and relapse (13). Previous studies assumed that, by stabilizing $\beta$-catenin in the nucleus, undifferentiated tumor state is maintained in WT $(3,4)$; work by Zhang et al $(10)$ on glioma furthermore suggested that this may be a consequence of elevated FOXM1 expression. Thus, we hypothesized that FOXM1 expression would be highest in those tumors of undifferentiated histology and thus adverse clinical outcome. In line with this hypothesis, significantly elevated FOXM1 expression levels were observed in tumors with blastemal and diffuse, as well as focal anaplastic, histopathology. Likewise, a trend towards decreased survival and increased incidence of relapse was revealed in those tumors of highest FOXM1 expression, possibly reflecting the association of FOXM1 and histopathological subtype.

In agreement with previous data showing inhibition of FOXM1 by doxorubicin (14), it was demonstrated in vivo that FOXM1 expression was low in tumors pretreated with doxorubicin ( $\mathrm{P}=0.009$; Mann-Whitney $\mathrm{U}$ ). While the precise
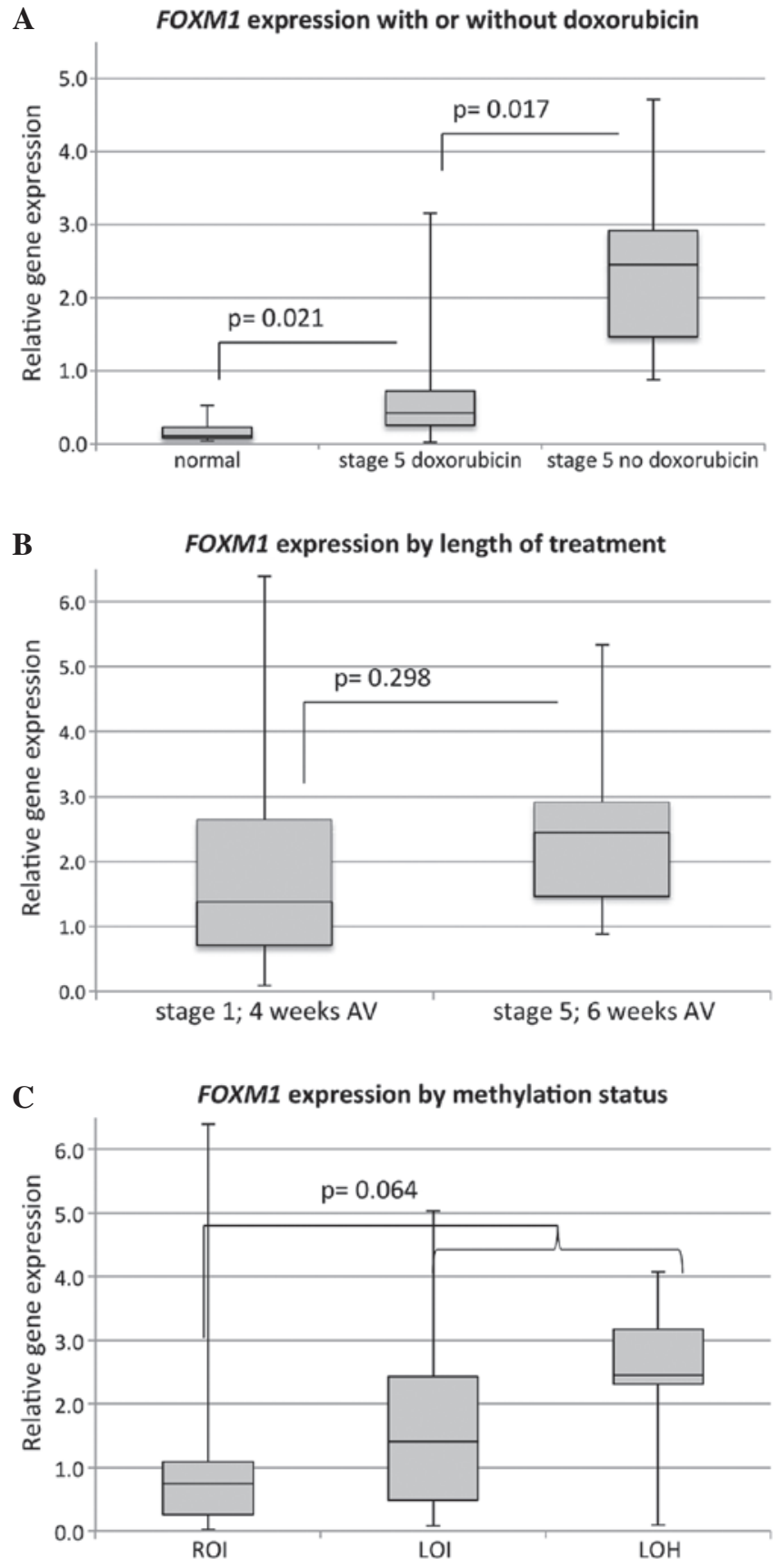

Figure 3. Differential FOXM1 expression is affected by doxorubicin administration but not by length of treatment. (A) Impact of preoperative doxorubicin treatment on FOXM1 expression in stage $\mathrm{V}$ tumors. (B) Prolongation of standard treatment duration alone (AV without the addition of doxorubicin) had no significant effect on FOXM1 expression (C) FOXM1 expression in tumors with ROI, LOI and $\mathrm{LOH}$ at the $I G F 2 / H 19$ locus $(\mathrm{P}=0.110)$. Statistical differences were calculated with the Mann-Whitney test. FOXM1, forkhead box M1; AV, vincristine and actinomycin D; ROI, retention of imprinting; LOI, loss of imprinting; LOH, loss of heterozygosity.

mechanism of doxorubicin action on FOXM1 is currently not understood, its proposed mechanism of action is via FOXO (14). Besides regulating cell proliferation, differentiation, DNA damage repair and apoptosis, FOXO is a known inhibitor of FOXM1 expression (14). Indeed, a diverse spectrum of anticancer drugs, including paclitaxel, lapatinib, gefitinib, imatinib and cisplatin, have been shown to derive their anticancer properties from acting on the FOXO/FOXMI axis (14). 
As a hypothesis-generating study, this work has some limitations that merit discussion, not the least of which is the comparatively small number of samples analyzed and the concomitant unusual distribution of gender. While this may limit the extent to which definite conclusions may be drawn from the work herein presented, it is certainly a motivation for future studies including a larger number of cases and ideally also samples of tumors resected by primary nephrectomy. This study has demonstrated that FOXM1 expression is suppressed by chemotherapy. While samples harvested at primary nephrectomy were unavailable for the present study under the current SIOP protocol, the requirement for assessing non-pretreated tissue for FOXM1 expression is one further argument favoring the extensive inter-institutional collaboration necessary in advancing the understanding of Wilms tumorigenesis.

In conclusion, the present data demonstrated significantly elevated FOXM1 expression levels in WT of high-risk histology. Based on two current models of WT ontogenesis $(3,4)$ as well as a glioma model (10), this may indeed reflect a causative association, where high $F O X M 1$ expression determines undifferentiated tumor state by stabilizing $\beta$-catenin in the nucleus. The present data on the association of FOXMI expression with prognosis may be interpreted as a reflection of this. Notably, the current study was the first to demonstrate in vivo that pretreatment with doxorubicin significantly reduces FOXMI expression independently of the increase in treatment length. Whether this latter observation has any future impact on improving outcome by targeting FOXM1 in those patients of high-risk histology remains the subject of studies to come.

\section{References}

1. Davidoff AM: Wilms tumor. Adv Pediatr 59: 247-267, 2012.
2. Dome JS, Fernandez CV, Mullen EA, Kalapurakal JA, Geller JI, Huff V, Gratias EJ, Dix DB, Ehrlich PF, Khanna G, et al: Children's oncology group's 2013 blueprint for research: Renal tumors. Pediatr Blood Cancer 60: 994-1000, 2013

3. Gadd S, Huff V, Huang CC, Ruteshouser EC, Dome JS, Grundy PE, Breslow N, Jennings L, Green DM, Beckwith JB and Perlman EJ: Clinically relevant subsets identified by gene expression patterns support a revised ontogenic model of Wilms tumor: A children's oncology group study. Neoplasia 14: 742-756, 2012.

4. Pode-Shakked N and Dekel B: Wilms tumor-a renal stem cell malignancy? Pediatr Nephrol 26: 1535-1543, 2011.

5. Hubertus J, Lacher M, Rottenkolber M, Müller-Höcker J, Berger M, Stehr M, von Schweinitz D and Kappler R: Altered expression of imprinted genes in Wilms tumors. Oncol Rep 25: 817-823, 2011.

6. Hubertus J, Zitzmann F, Trippel F, Müller-Höcker J, Stehr M, von Schweinitz D and Kappler R: Selective methylation of CpGs at regulatory binding sites controls NNAT expression in Wilms tumors. PLoS One 8: e67605, 2013.

7. Katoh M, Igarashi M, Fukuda $\mathrm{H}$, Nakagama $\mathrm{H}$ and Katoh $\mathrm{M}$ : Cancer genetics and genomics of human FOX family genes. Cancer Lett 328: 198-206, 2013.

8. Halasi M and Gartel AL: Targeting FOXM1 in cancer. Biochem Pharmacol 85: 644-652, 2013.

9. Gong $\mathrm{A}$ and Huang S: FoxM1 and Wnt/ $\beta$-catenin signaling in glioma stem cells. Cancer Res 72: 5658-5662, 2012.

10. Zhang N, Wei P, Gong A, Chiu WT, Lee HT, Colman H, Huang H, Xue J, Liu M, Wang Y, et al: FoxM1 promotes $\beta$-catenin nuclear localization and controls Wnt target-gene expression and glioma tumorigenesis. Cancer Cell 20: 427-442, 2011.

11. de Kraker J, Graf N, van Tinteren H, Pein F, Sandstedt B, Godzinski J and Tournade MF; SIOP: Reduction of postoperative chemotherapy in children with stage I intermediate-risk and anaplastic Wilms' tumour (SIOP 93-01 trial): A randomised controlled trial. Lancet 364: 1229-1235, 2004.

12. Pfaffl MW: A new mathematical model for relative quantification in real-time RT-PCR. Nucleic Acids Res 29: e45, 2001.

13. Vokuhl C, Vogelgesang W, Leuschner I, Furtwängler R, Graf N, Gessler M, Dörner E and Pietsch T: 1q gain is a frequent finding in preoperatively treated Wilms tumors, but of limited prognostic value for risk stratification in the SIOP2001/GPOH trial. Genes Chromosomes Cancer 53: 960-962, 2014.

14. Wilson MS, Brosens JJ, Schwenen HD and Lam EW: FOXO and FOXM1 in cancer: The FOXO-FOXM1 axis shapes the outcome of cancer chemotherapy. Curr Drug Targets 12: 1256-1266, 2011. 